\title{
Plan of NNbar experiment at the WWR-M reactor
}

\author{
Alexey Fomin ${ }^{1, a}$, Anatolii Serebrov ${ }^{1}$, Mikhail Chaikovskii ${ }^{1}$, Oleg Zherebtsov ${ }^{1}$, Aleksandr Murashkin ${ }^{1}$, \\ and Elena Golubeva ${ }^{2}$ \\ ${ }^{1}$ NRC "Kurchatov Institute" - PNPI, Gatchina, Russia \\ ${ }^{2}$ Institute for Nuclear Research, Russian Academy of Sciences, Moscow, Russia
}

\begin{abstract}
We propose an experiment to search for neutron-antineutron oscillations based on the storage of ultracold neutrons (UCN) in a material trap. This experiment will be competitive due to a new UCN source which is under construction at the NRC "Kurchatov Institute" - PNPI. It is expected to provide UCN densities by 2-3 orders of magnitude higher than at existing sources. The sensitivity of our experiment mostly depends on the trap size and the stored number of UCN. Simulations show that this can be increased by $\sim 10-40$ times compared to the best previous experiment, dependent upon a model of neutron reflection from walls. The project currently includes designs of the UCN chamber, magnetic shielding and the detector system, as well as simulations of neutron storage and detection of annihilations events.
\end{abstract}

\section{Introduction}

An oscillation between neutron and antineutron is a hypothetical process in which a neutron spontaneously transforms into an antineutron. As neutrons and antineutrons are both electrically neutral particles, only conservation of baryon number forbids this process. However, according to A. Sakharov [1], baryon number violation is one of three conditions which are essential to explain the observed matter-antimatter or baryon number asymmetry of the Universe.

There are two methods to search for $n \bar{n}$ oscillations, either using free neutrons or neutrons bound in nuclei. The probability of the transition is given by

$$
P(\bar{n}, t)=\frac{\delta m^{2}}{\delta m^{2}+\Delta E^{2}} \sin ^{2}\left(\sqrt{\delta m^{2}+\Delta E^{2}} \Delta t\right) .
$$

Therein, $\delta m=\left(\tau_{n \bar{n}}\right)^{-1}$ is the off-diagonal term in the effective Hamiltonian, and $B<10 \mathrm{nT}$ is half of the energy gap between the $n$ and $\bar{n}$ states due to nuclear and/or electromagnetic fields. For neutrons in nuclei, the (nuclear-model dependent) energy gap is wide and hence the oscillation is strongly suppressed due to the nuclear environment. For free neutrons only the external magnetic field and the quality of the vacuum matter. Experiments with free neutrons only investigate baryon number violation with an antineutron in the final state, whereas measurements with nuclei are also sensitive to other baryon number violating processes. The two approaches are thus complementary, which motivates experiments of both types. The latest one using free neutrons was finished in 1991 and established the highest limit on the oscillation time of the free neutron [2]. Experiments using neutrons bound in nuclei being carried out in underground laboratories reach similar limits [3].

According to Eq. (1) the probability of oscillations would be maximum if $t=0.4 \mathrm{~s}$. Also, if $\tau_{n \bar{n}} \gg t$ (which

a e-mail: fomin_ak@pnpi.nrcki.ru is always the case for the free-neutron approach since the neutron lifetime is much smaller than the oscillation time), then Eq. (1) can be simplified to:

$$
P(\bar{n}, t)=\sin ^{2}(\delta m t) \approx\left(\frac{t}{\tau_{n \bar{n}}}\right)^{2} .
$$

In the experiment this approximation can be used if the socalled "quasi-free" condition $\Delta E \cdot t \ll 1$ is satisfied [4]. The standard approach to search for free $n \bar{n}$ oscillations is to project a beam of slow neutrons onto a target of a material with a good annihilation cross-section, maintaining the quasi-free condition throughout the flight path of the beam. In such experiments $t$ is time of neutron propagation within the quasi-free condition region.

Here we discuss a different free-neutron approach using ultracold neutrons (UCN) stored in a material trap. The annihilation target is the whole inner surface of the trap, and $t$ is the time of free propagation between UCN collisions with the trap walls which depends on the trap size [5-8]. The quasi-free condition has to be maintained within the UCN trap. The goal of the study presented in this paper is to estimate the efficiency of the UCN approach to search for $n \bar{n}$ oscillation.

\section{UCN trap and the quasi-free condition}

The quantitative parameter we try to determine and compare with existing experiments is the sensitivity of the proposed experimental apparatus:

$$
S=N t^{2} \varepsilon .
$$

Therein, $N$ is the total frequency of neutron collisions with the annihilation target, $t$ is the mean time of free neutron propagation to target, and $\varepsilon$ is the efficiency of annihilation detection (hence $\varepsilon<1$ ). The mean number of antineutrons is $\langle\bar{n}\rangle=\left(S / \tau_{n \bar{n}}^{2}\right) T$, where $T$ is the total time of data collection. The product $N t^{2}$ is a figure 
of merit characterizing the UCN source and the trap. This parameter is to be estimated first in this project. $N$ depends on the mean total number of $\mathrm{UCN}$ in the trap. It is determined by the UCN source intensity and the UCN storage lifetime; $t$ depends on the trap's size and shape.

The trap size is limited by the space available in the reactor hall at the WWR-M facilities and by the total mass of the apparatus. Special Monte-Carlo simulations were performed to estimate values for $t$ possible under these conditions. Basically, the bigger the trap is, the longer the propagation period. However, it becomes ineffective to increase the trap beyond a certain size because acceleration of the neutron due to gravity combined with a limited neutron optical wall potential limits the time of free propagation. As a result of the simulations, we decided for the trap to be a horizontal cylinder with a diameter of $2 \mathrm{~m}$ and a length of $4 \mathrm{~m}$ and found $t=0.4 \mathrm{~s}$ for this geometry.

While the neutron storage lifetime cannot be made longer than the beta decay lifetime of the neutron, it can still reach values exceeding $800 \mathrm{~s}$ in well-designed traps. We plan to achieve good UCN storage properties by using a copper trap covered with a thin Ni-Mo layer.

Calculations show that at currently existing UCN sources $N t^{2}$ cannot be larger than in beam experiments. However, a new high-intensity UCN source currently under construction at the WWR-M reactor at PNPI, Gatchina [9] (see also these proceedings) will increase the UCN intensity by two orders of magnitude, as shown by flux simulations [10]. Compared to the best previous beam experiment [2] the UCN experiment will then have a 10-40 times higher sensitivity and thus becomes competitive. As it is yet unknown if ultracold antineutrons can be reflected from the trap wall, the quoted range relates to the uncertainty of corresponding models [5] (10 times increase in sensitivity corresponds to zero reflections of antineutrons).

To provide a high vacuum for neutron propagation, the trap will be placed in an aluminium tank. The inner cavity of the tank will be pumped out by turbo-molecular pumps. The wall thickness of the tank influences the probability of annihilation event registration by stopping emitted particles. Therefore it should be as thin as possible, yet sustaining the outer pressure. Simulations of the detector efficiency revealed that the chosen wall thickness of $8 \mathrm{~mm}$ reduces the number of potentially detectable events by $5 \%$.

The antiparallel orientation of the magnetic moments of the neutron and the antineutron causes an energy difference $2 \Delta E$ in the external magnetic field (see Eq. (1)). In order to fulfill the quasi-free condition, it is thus necessary to magnetically shield the oscillation region. In the experiment implemented at a cold neutron beam at the ILL [2], the Earth's field was suppressed to a level $B<$ 10nT. In our setup, the mean time of flight of a neutron is larger $(t=0.4 \mathrm{~s})$, and hence we need to suppress it to $B \approx 1 \mathrm{nT}$. Calculations show that this requires a two-layer magnetic screen. As for the vacuum chamber, an additional requirement is that the screens have to be sufficiently thin not to suppress the detection efficiency. It was found that two cylindrical screens with $1.5 \mathrm{~mm}$ thickness each made of $\mu$-metal fulfil both requirements. To improve the axial shielding factor, which is usually an order of magnitude smaller than the transverse one, a solenoid will be used for active field compensation. In the present model, the inner screen has a length of $4950 \mathrm{~mm}$ and a diameter of $2730 \mathrm{~mm}$, with a gap of $97 \mathrm{~mm}$ between the screens.

\section{Detector and registration efficiency}

The registration of antineutrons appearing due to oscillation in the trap relies on an annihilation at the trap wall. Annihilation with a nucleon results in an emission of several charged and neutral $\pi$ mesons. However, as this occurs in a nuclear environment, $\pi$ mesons first propagate through the primary nucleus where the annihilation took place, followed by nuclear de-excitation. In our present model we use Ni-Mo coating of the copper trap surface. Therefore, annihilation of antineutrons occurs either in a $\mathrm{Ni}$ or a Mo nucleus. The final state contains $\pi$ mesons along with additional particles: nucleons and heavier fragments of the nucleus, such as alpha particles, deuterons, tritons, etc. The latter come with large abundance: protons are emitted in $80.5 \%$ of events, neutrons in $82.5 \%$, alpha particles in $30 \%$, while other particles occur less abundantly. Below we will call "initial event" the annihilation together with all subsequent processes in the primary nucleus, and "initial particles" all those particles emitted from the initial event.

Special simulations were carried out to model the nuclear processes associated with annihilation events in $\mathrm{Ni}$ and Mo nuclei (for a more detailed description of the model see Ref. [11]). We used the generated events to develop the detector system and estimate the detector efficiency. At the current stage of this project, we are trying to understand the general features of the processes in the apparatus. As simulations can quickly become very time consuming, we limited the number of events to $7.5 \times 10^{4}$. An annihilation releases roughly $2 \mathrm{GeV}$ of energy in the form of $\pi$ mesons and kinetic energy of all other particles in the final state. The total momentum is almost zero, corresponding to a roughly spherically symmetric momentum and energy deposition of particles emitted from the trap surface. These facts provide trigger conditions for registration.

For the selection of appropriate detector configurations and an estimation of the detection sensitivity we used an MC model of the detector created in GEANT4. We consider the scheme in which the trap is placed into the vacuum vessel, the vessel into the magnetic screen, and the detector surrounds the screen (see Fig. 1). In this scheme, the magnetic screen and the region inside it is a "blind area" of our apparatus - below we call it also the "inner region" - and we call the "outer region" the active area of the detector. In the first step of our simulation, we considered the propagation of particles through the inner region. From this analysis we could draw several conclusions:

- A significant part of relatively low energy particles (numbers will be given further below) will be stopped in the blind area, notably protons and heavier nuclear fragments.

- Propagation through blind areas changes the initial directions of the particles.

- Initial particles subsequently interact with the material of the trap, the vacuum vessel, and the magnetic screens, resulting in emission of charged and neutral 
"secondary" particles. Charged high-energy particles emitted are mostly electrons and positrons from the interaction of gamma quanta with matter.

Charged pions - The average energy of charged pions which leave the primary nucleus after the intranuclear cascade process is $240 \mathrm{MeV}$. While this result is based only on the model of annihilation, our GEANT4 simulations also provide results for the propagation of these particles through detector materials. It is found that $(69 \pm 2) \%$ of the charged pions are able to leave the magnetic screen. The average angle between the initial direction and the direction after leaving the second layer of the screen is $(6.7 \pm 0.2)^{\circ}$. The average energy of charged pions which leave both layers of the magnetic screen is $(282 \pm 3) \mathrm{MeV}$, whereas the average energy of those which do not is $(142 \pm 2) \mathrm{MeV}$. This indicates that, as expected, lower energy particles mostly cannot leave the blind area.

Protons - For protons emitted from the initial event, only $(8 \pm 1) \%$ can leave the screen and the average deflection is $(4.4 \pm 0.1)^{\circ}$. Their average initial energy is $90 \mathrm{MeV}$. The average energy of the protons which reach the outer region is $(185 \pm 5) \mathrm{MeV}$, whereas for stopped protons it is $(32 \pm 2) \mathrm{MeV}$. Hence, only protons with higher energies can create a signal in the detector.

In comparison with cold-neutron beam experiments, the advantage of our approach is the absence of background from inside the trap. Electrons and protons from the decay of UCN have too low energy to pass the trap wall, while the antineutrino leaves the apparatus without any interaction. Another considerable advantage is the size of the apparatus. The whole neutron path is located within a relatively compact region. The price to pay, however, is having the annihilation target in the propagation region and the target being distributed along all the surface of the trap. Taking into account the advantages and disadvantages of our approach, and summarizing the result of the first stage of these simulations, we conclude that the best selection criterion for annihilation events should be built on propagation of high-energy charged particles from the trap to the outer region.

Based on these results, we created a model of the detection system. The main component is a hodoscope made of two layers of $20 \mathrm{~mm}$ thick and $210 \mathrm{~mm}$ wide plastic scintillator plates with photomultiplier tubes (PMT's) at both ends of each plate. Such an event recording system is required to have time resolution of at least $2 \mathrm{~ns}$ to distinguish the flight direction of particles either from the inner to the outer region, or vice versa.

As the whole apparatus will be located in the reactor hall, the main external background components will be due to neutrons, gamma quanta, and cosmic rays. One problem is that there are charged particles which can penetrate the trap and imitate the sought signal. Another problem is that spurious hodoscope signals may occur due to accidental coincidences of background signals in both hodoscope layers. To exclude the latter, we add a tracker system for direct observation of the particle tracks. This will also help us to exclude signals from cosmic background with a signature of two correlated signals, the first propagating from the outer to the inner layer and the second vice versa. The main particles to register are charged $\pi$ mesons and protons from initial events as well as electrons and positrons from interactions of gamma quanta with the trap

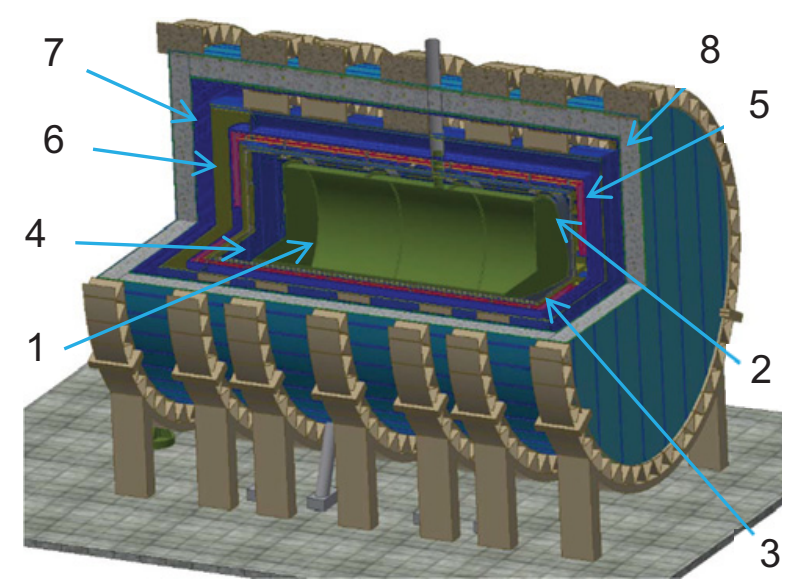

Figure 1. Schematic of the setup with a $90^{\circ}$ cutout to show the inner structure of the trap. 1: UCN trap, 2: vacuum vessel, 3: magnetic screen, 4: inner tracker, 5: inner hodoscope layer, 6: middle tracker, 7: outer hodoscope layer, 8: outer tracker.

and other parts of the apparatus. All of these will leave tracks in the wire chambers.

The planned tracker system consists of wire chamber blocks arranged in three concentric cylinders around the trap. Each block is $20 \mathrm{~mm}$ thick and $304 \mathrm{~mm}$ wide, filled with a mixture of $70 \% \mathrm{Ar}, 25 \% \mathrm{CO}_{2}$, and $5 \%$ additional gases. The innermost tracker layer will be placed between the magnetic screen and the vacuum vessel, the middle layer between hodoscope layers and the outer layer will be outside the hodoscope system.

The two inner tracker systems will consist of only two layers of wire chambers. As their purpose is to identify hodoscope signals belonging to particles propagating through the whole system, we want to minimize their influence on trajectories. Additional layers would result in additional energy loss and deflection. The two layers are arranged as such to leave no space between the chambers and hence all particles have to go through at least one of the blocks.

The outer tracker will consist of several layers of wire chamber blocks enforced by $1-2 \mathrm{~mm}$ thick lead cylinders and $2.5 \mathrm{~mm}$ thick aluminum cylinders between tracker layers. It serves two purposes: 1) for most initial particles a signal in several layers will result in better tracking and exclusion of spurious hodoscope signals; 2) it provides an active veto for protection of the inner detector system from backgrounds. The number of layers is still under consideration. Currently, we use a model with 12 layers. The scheme of the whole setup is shown in Fig. 1.

In the second stage of our MC simulations, we investigated particle propagation through the whole detector system, using the generation of initial events as discussed above. The goal was to find suitable criteria for registration of an annihilation event.

In total, $(55 \pm 2) \%$ of the initial $\pi^{+}$and only $(3.5 \pm 0.3) \%$ of the initial protons provide signals in all three tracker systems. The simulated energy distributions reveal that a significant part of the initial energy becomes dissipated in the "blind area", notably in the case of protons. Most of the $\pi^{ \pm}$mesons penetrate several rows of the outer tracker. In the current model with 12 layers, about $50 \%$ of the $\pi^{ \pm}$mesons which leave signals in 


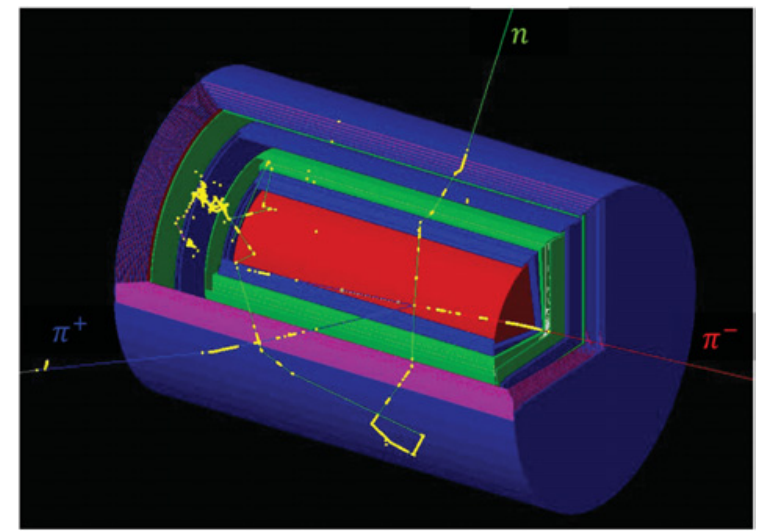

Figure 2. GEANT4 model of the detection system and an illustrative annihilation event that has is passed the criteria for event selection. Red cylinder: vacuum vessel, blue cylindrical layers: tracker layers of inner, middle and outer trackers, green: inner and outer hodoscope layers.

all tracker systems pass through all layers of the outer tracker. Protons more often stop in outer tracker due to energy dissipation, but a significant portion of them still penetrate several rows. Most secondary particles stop in the outer tracker. These results can in principle be used to distinguish particles by the amount of layers in which the signal is obtained, but at the current stage of the project, we did not study the impact of such additional criteria on their capacity to reduce backgrounds.

Also in this second stage of MC simulations, we calculated characteristic time intervals. We found that the average time of flight between hodoscope layers for the $\pi \pm$ mesons is $(2.9 \pm 0.2) \mathrm{ns}$ with a minimum of about $2 \mathrm{~ns}$. This defines the required level of time resolution of the hodoscope system, which is perfectly achievable at the present state of technology. At the same time, the maximum time of flight between layers can reach $30 \mathrm{~ns}$, which approximately defines the time interval within which signals from a single event may occur. Clearly, the longer the time window, the more background events will be recorded; if the window is too short, the sensitivity to true events will decrease. Thus, the time interval within which we search for a true signal will have to be carefully selected. Average time differences are in the order of $5 \mathrm{~ns}$ and time intervals above $20 \mathrm{~ns}$ occur very rarely. In ongoing simulations, we have been using a time window of $25 \mathrm{~ns}$, which can in the future be adjusted to the final detection system when actually implemented. The current GEANT4 model does not take into consideration some of the construction details of the setup, because its main purpose has been to guide the development of the detector system. The model in its present form is sufficient to provide a general estimate of the detector efficiency. We expect that a refined model to be constructed in the future will lead only to small modifications of the results. For illustration, Fig. 2 shows one of the annihilation events simulated in the GEANT4 model discussed, and which passes the criteria for event selection.

Summarizing all MC calculations, we have made a conclusion about event selection criteria and estimated the detector sensitivity. We consider the occurrence of two simultaneous signals in the hodoscope in coincidence with signals in all three trackers as the most suitable criteria for an annihilation event selection. To test this trigger condition, we simulated $4 \times 10^{4}$ annihilation events and obtained a detection efficiency of $\varepsilon=(68 \pm 2) \%$. Here detector efficiency is the percent of annihilation events which passed selection.

We are also considering several additional criteria for the distinction of annihilation events from backgrounds, in case the chosen trigger condition will be insufficient. The necessity of this measure will be revealed only after the start of measurements with our constructed detector. We consider three possibilities, which all have particular issues that cannot be addressed at the current stage of this project:

- Use a correlation of the energy of emitted particles in the initial event with the number of signals in the trackers. This requires a careful calibration of the detector and study of the signals of background events. The problem is that the energy spectrum of initial events is spread by dissipation of energy and losses of particles in the trap and the vacuum vessel, which makes it difficult to distinguish signals of annihilation events from background events.

- Use the fact that distributions of energy and momentum are spherically symmetric for the initial events. The size and form of the trap and the fact that annihilation can occur at any point on the surface of the trap make this criterion very difficult to use. We plan to carry out additional MC calculations to determine a selection criterion connected to the distribution of signals in the detector. Unfortunately, it also requires estimations of background influence.

- Use the reconstruction of the event vertex, which has to be at the surface of the trap. As mentioned above, the direction of particles significantly changes in the blind area and the inner tracker is separated from the event vertex by at least $50 \mathrm{~cm}$. Hence, we will not be able to reconstruct the vertex with a precision better than several centimeters, but this could still be enough to be useful.

The last criterion is related to the question of selection of initial particle signals and secondary particle signals. As mentioned above, secondary particles mostly cannot pass several layers of the outer tracker, while initial $\pi^{ \pm}$ mesons have $50 \%$ probability to penetrate the whole outer tracker. This suggests another selection criterion - to use only tracks with propagation through at least 6 layers of the outer tracker and a reconstructed vertex to be in the vicinity of the trap wall. Employment of such a criterion would, however, drop the detector efficiency to $30-35 \%$.

For all additional criteria, as well as for advanced calculations, we need to measure background conditions at the experimental site and complete calibrations of the detectors. All of this can only be completed after the construction of the detector systems as well as the active and passive magnetic shielding.

The development of the NNbar experiment was supported by Russian Foundation for Basic Research, grant no. 16-02-00778a. The development of the UCN source at the reactor WWR-M was supported by Russian Science Foundation, grant no. 14-2200105 . 


\section{References}

[1] A.D. Sakharov, JETP Lett. 5, 24 (1967)

[2] M. Baldo-Ceolin, P. Benetti, T. Bitter et al., Z. Phys. C 63, 409 (1994)

[3] D.G. Phillips et al., Phys. Rep. 612, 1 (2016)

[4] R.N. Mohapatra, R. E. Marshak, Phys. Lett. B 94, 183 (1980)

[5] A.P. Serebrov, A.K. Fomin, Yu. A. Kamyshkov, Tech. Phys. Lett. 42, 99 (2016)
[6] A.K. Fomin, A.P. Serebrov, O.M. Zherebtsov et al., J. Phys. Conf. Ser. 798, 012115 (2017)

[7] A. Fomin, PoS INPC2016, 189 (2017)

[8] A.K. Fomin, A. P. Serebrov, O.M. Zherebtsov et al., KnE Energy \& Physics 3, 109 (2018)

[9] A.P. Serebrov, Crystallogr. Rep. 56, 1230 (2011)

[10] A.P. Serebrov, A.K. Fomin, Tech. Phys. 85, 136 (2015)

[11] E.S. Golubeva, J.L. Barrow, C.G. Ladd, Phys. Rev. D 99, 035002 (2019) 